\title{
Digital Image Filtering Techniques- A survey
}

\author{
Sakshi Mahajan, Navneet Bawa \\ Department of Information Technology , ACET, Amritsar (Pb.) India \\ sakshimtech@gmail.com \\ Department of Computer Science, ACET, Amritsar (Pb.) India \\ bawa.navneet@gmail.com
}

\section{ABSTRACT}

This paper presents a review on digital image filtering techniques. The main emphasis is on median filtering and its extended versions like hybrid median filtering, relaxed median filtering etc. It is found that still median filtering demands some sort of enhancements as it is best for salt and pepper noise only. By conducting a survey suitable gaps are found in existing literature. In the end comparison table is also drawn among the existing techniques.

\section{Indexing terms/Keywords}

Salt and pepper noise, Median filter, Smoothing and Sharpening.

\section{Academic Discipline And Sub-Disciplines}

Digital Image Processing is an important research area.The techniques developed in this area are useful in various day to day operations like in medical sciences, weather forecasting,remote sensing, robotics, photography and intelligent systems.

\section{SUBJECT CLASSIFICATION}

Digital Image Processing is a method which uses various computer algorithms in order to process digital image.It is the field in which various numerical computation are performed on a digital image in order to enhance or restore the original image.

\section{TYPE (METHOD/APPROACH)}

In this research paper various filtering techniques are described that are used in spatial domain which helps in image restoration and enhancement .

\section{Council for Innovative Research}

Peer Review Research Publishing System

Journal: INTERNATIONAL JOURNAL OF COMPUTER AND TECHNOLOGY

Vol 10, No. 6

editor@cirworld.com

www.cirworld.com, member.cirworld.com 


\section{I.Introduction}

In image processing, noise reduction and restoration of image is expected to improve the qualitative inspection of an image and the performance criteria of quantitative image analysis techniques. Digital image is inclined to a variety of noise which affects the quality of image. The main purpose of de-noising the image is to restore the detail of original image as much as possible. The criteria of the noise removal problem depend on the noise type by which the image is corrupting. In the field of reducing the image noise several types of linear and non linear filtering techniques have been proposed. Different approaches for reduction of noise and image enhancement [1] have been considered, each of which has their own limitation and advantages.

Image de-noising is a vital image processing task i. e. as a process itself as well as a component in other processes. There are many ways to de-noise an image or a set of data and methods exists. The important property of a good image denoising model is that it should completely remove noise as far as possible as well as preserve edges. Traditionally, there are two types of models i. e. linear model and non-liner model. Generally, linear models are used. The benefits of linear noise removing models is the speed and the limitations of the linear models is, the models are not able to preserve edges of the images in a efficient manner i. e. the edges, which are recognized as discontinuities in the image, are smeared out. On the other hand, Non-linear models [2] can handle edges in a much better way than linear models.

\section{Applications of filtering}

There are various application of filtering that can help in image enhancement and restoration. Some of them are described below

\section{A. Noise Smoothing}

Linear filters are good for smoothing most types of noise; however this is at the expense of edge sharpness and fine detail. These limitations can be overcome by using trimmed filters. The MEDIAN filter is good for smoothing noise while preserving edges.

\section{B. Edge Enhancements}

Edge enhancement is often achieved by steepening the edge slope. When linear filters are used for this, it results on edges which are already sharp. Nonlinear filter work more reasonably well in preserving edges with the help of various order statistics filter.

\section{Edge Detection}

Linear edge detection filters [3] can be made to detect edges in one particular direction or in all directions at once. Many nonlinear filters have been designed to improve edge direction in the presence of noise.

\section{Types of Noises}

The main source of noise in digital images arises during image acquisition (digitization) or during image transmission. The performance of image sensor is affected by variety of reasons such as environmental condition during image acquisition or by the quality of the sensing element themselves. For instance, during acquiring images with CCD camera, sensor temperature and light levels are major factors that affecting the amount of noise in the image after the resulting. Images are corrupted while during transmission of images. The principal reason of noise is due to interfering in the channel which is used for the images transmission. We can model a noisy image as follows:

$$
C(x, y)=A(x, y)+B(x, y)
$$

Where $A(x, y)$ is the original image pixel value and $B(x, y)$ is the noise in the image and $C(x, y)$ is the resulting noise image [4].

A. Uniform Noise: The uniform noise cause by quantizing the pixels of image to a number of distinct levels is known as quantization noise. It has approximately uniform distribution. In the uniform noise the level of the gray values of the noise are uniformly distributed across a specified range.

B. Gaussian Noise or Amplifier Noise: This noise has a Probability Density Function of the normal distribution. It is also known as Gaussian distribution. It is a major part of the read noise of an image sensor that is of the constant level of noise in the dark areas of the image

C. Salt and Pepper Noise: The salt-and-pepper noise[4] are also called shot noise, impulse noise or spike noise that is usually caused by faulty memory locations, malfunctioning pixel elements in the camera sensors, or there can be timing errors in the process of digitization. For 8-bit image the typical value for 255 for salt-noise and pepper noise is 0.

D. Rayleigh Noise: Radar range and velocity images typically contain noise that can be modeled by the Rayleigh distribution.

E. Gamma Noise: The noise can be obtained by the low-pass filtering of laser based images. 


\section{Filters}

Filtering in an image processing is a basis function that is used to achieve many tasks such as noise reduction [5], interpolation, and re-sampling. Filtering image data is a standard process used in almost all image processing systems. The choice of filter is determined by the nature of the task performed by filter and behavior and type of the data. Filters are used to remove noise from digital image while keeping the details of image preserved is a necessary part of image processing. Filters can be described by different categories

\section{A. Spatial Domain Filtering}

The Spatial Domain is a domain (the plane) where a digital image is defined by spatial coordinates of its pixels. The spatial domain processes can be represented by the following expression $g(x, y)=T[f(x, y)]$ where $f(x, y)$ is the input image, $g(x$, $y$ ) is the output image and $T$ is an operator defined over a local neighborhood of pixel with the coordinates( $x, y)$. There are two types of spatial filters.

\section{i. Smoothing Filters}

\section{(a) Low Pass Spatial Filtering}

The simplest form of spatial filtering is a uniform neighborhood averaging. This is accomplished using a spatial mask of all ones. The effect of low pass filtering is to make edges more diffuse and low contrast.

\section{(b) Median Filtering}

Median filtering [6] is done one neighborhood at a time; however the mask that it uses is not a linear function. A median filter [7] replaces the pixel with the median of the neighborhood. This is useful in removing noise from a single image. The median filter [11] does this by removing large noise spikes from the image.

\section{ii. Sharpening Filters}

\section{(a) High Pass Spatial Filtering}

The effect that high pass filters have on an image is exactly opposite that of low pass filters. The primary goal of low pass filtering is to highlight detail or to enhance lost detail due to blurring or faults in image acquisition. This is achieved using a mask having a positive value in its center location and negative coefficients in the rest. A high pass [13] filtered image may be computed also as the difference between the original image and a low pass filtered version of the image.

\section{(b) High Boost Filtering}

In this a blurred image is subtracted from original to get unsharp mask image, then we add a multiple of unsharp mask to original to get sharpened image. Example of high boost filtering is Unsharp Masking.

\section{B. Frequency Domain Filtering}

In this techniques are based on modifying the spectral transform of an image. It transforms the image to its frequency representation and performs image processing and after that it computes inverse transform back to the spatial domain. High frequencies correspond to pixel values that change rapidly across the image (e. g. text, texture, leaves, etc.). Strong low frequency components correspond to large scale features in the image (e. g. a single, homogenous object that dominates the image).

\section{(a) Smoothing Frequency Domain Filters}

Smoothing is achieved in the frequency domain by dropping out the high frequency components. The basic model for filtering is: $\mathrm{G}(\mathrm{u}, \mathrm{v})=\mathrm{H}(\mathrm{u}, \mathrm{v}) \mathrm{F}(\mathrm{u}, \mathrm{v})$

Where $F(u, v)$ is the Fourier transform of the image being filtered and $H(u, v)$ is the filter transform function. Low pass filters - only pass the low frequencies, drop the high ones.

\section{(b) Sharpening Frequency Domain Filters}

Edges and fine detail in images are associated with high frequency components.

High pass filters - only pass the high frequencies, drop the low ones. High pass frequencies are precisely the reverse of low pass filters, so: $\operatorname{Hhp}(u, v)=1-H l p(u, v)$

\section{Literature Survey}

S. Esakkirajan et al. (2011) [1] described a new algorithm Modified Decision Based Unsymmetric Trimmed Median Filter (MDBUTMF) which gives better performance in comparison with existing noise removal algorithms in terms of PSNR and IEF. The performance of the algorithm has been tested at different noise densities on both gray-scale and color images. Even at high noise density levels the MDBUTMF gives better results in comparison with other existing algorithms. Both visual and quantitative results are demonstrated. The algorithm is effective for salt and pepper noise removal in images at high noise densities. 
Priyanka Kamboj et al. (2013) [2] described that Enhancement of a noisy image is necessary task in digital image processing. Filters are used best for removing noise from the images. Various types of noise models and filters techniques have been described. Filters techniques are divided into two parts linear and non-linear techniques. After studying linear and non-linear filter each of have limitations and advantages.

Shanmugavadivu et al. (2012) [3] proposed a filter which is more effective in restoring the images corrupted with fixedvalue impulse noise. As the proposed filter is computationally simple, the restoration rate is faster. This filter finds application in eliminating noise from various scanning images, used in the study of surface morphology, because these images are invariably degraded by fixed value impulse noise.

Shanmugavadivu P et al. (2011) [4] defined a newly devised noise filter namely, Adaptive Two-Stage Median Filter (ATSM) to denoise the images corrupted by fixed-value impulse noise. The performance of the proposed filter is proved to be better in terms of Peak Signal-to-Noise Ratio and human visual perception. This filter is effectual in denoising the highly corrupted image.

K. S. Srinivasan et al. (2007) [5] discussed a new decision-based algorithm for restoration of images that are highly corrupted by impulse noise. The new algorithm shows significantly better image quality than a Standard Median Filter and various nonlinear filters. The proposed method, unlike other nonlinear filters, removes only corrupted pixel by the median value or by its neighboring pixel value.

V. Jayaraj et al. (2010) [6] described the new method which introduces the concept of substitution of noisy pixels by linear prediction prior to estimation. A novel simplified linear predictor is developed for this purpose. The objective of the scheme and algorithm is the removal of high-density salt and pepper noise in images.

K. Aiswarya et al. (2010) [7] described a new algorithm to remove high-density salt and pepper noise using modified sheer sorting method. The new algorithm has lower computation time when compared to other standard algorithms. Results of the algorithm are compared with various existing algorithms and it is proved that the new method has better visual appearance and quantitative measures at higher noise densities.

Gnanambal llango et al. (2011) [8] introduced various hybrid filtering techniques for removal of Gaussian noise from medical images. The performance of Gaussian noise removing hybrid filtering techniques is measured using quantitative performance measures such as RMSE and PSNR. The experimental results indicate that the Hybrid Max Filter performs significantly better than many other existing techniques and it gives the best results after successive iterations. The method is simple and easy to implement.

P. E. Ng et al. (2006) [9] proposed a novel switching median filter incorporating with a powerful impulse noise detection method for effectively denoising extremely corrupted images. To determine whether the current pixel is corrupted, the algorithm first classifies the pixels of a localized window, centering on the current pixel, into three groups-lower intensity impulse noise, uncorrupted pixels, and higher intensity impulse noise.

Zinat Afrose (2012) [10] described a method to remove Salt \& pepper, Gaussian and Speckle noise from compound images using median filter, relaxed median filter, wiener, centre weighted median and averaging filter . The performance of the different filters with the applied noises using compound images are compared and analyzed according to PSNR value.

Table 1:Summary of Literature Survey

\begin{tabular}{|l|l|l|}
\hline Ref. No & Technique & \multicolumn{1}{|c|}{ Results } \\
\hline$[1]$ & $\begin{array}{l}\text { Modified Decision Based Unsymmetric } \\
\text { Trimmed Median Filter }\end{array}$ & Improved PSNR and IEF \\
\hline$[2]$ & Hybrid Filtering & Improved PSNR and MSE \\
\hline$[3]$ & Laplace Equation Adaptive Median Filter & Improved PSNR \\
\hline$[4]$ & Adaptive Two stage Median Filter & Improved PSNR \\
\hline$[5]$ & $\begin{array}{l}\text { Decision Based Algorithm removes only } \\
\text { corrupted image }\end{array}$ & Improved PSNR \\
\hline$[6]$ & $\begin{array}{l}\text { Switching based Median filter technique } \\
\text { Improved PSNR,MSE, Iow } \\
\text { computational Time }\end{array}$ \\
\hline$[7]$ & $\begin{array}{l}\text { Modified Sheer Sorting method PSN, lower } \\
\text { Hybrid Filtering }\end{array}$ & $\begin{array}{l}\text { Improved PSproved RMSE } \\
\text { computational Time }\end{array}$ \\
\hline$[9]$ & $\begin{array}{l}\text { Boundary Discriminative Noise Detection } \\
\text { Switching median filter }\end{array}$ & $\begin{array}{l}\text { Improved PSNR,Simple } \\
\text { real time application }\end{array}$ \\
\hline$[10]$ & Relaxed Median Filter & Improved PSNR \\
\hline
\end{tabular}




\section{Conclusion and Future work}

Image restoration is the process of eliminating or reducing noise from a degraded image with an objective to recover, the original image. Noise is a quality degradation factor that is measured as unwanted/unrelated information present in the image Several non linear filters have been proposed for the restoration of images contaminated by salt and pepper. The Modified Decision Based Unsymmetric Trimmed Median Filter (MDBUTMF) algorithm removes impulse noise at high noise density and gives better Peak Signal-to-Noise Ratio (PSNR) and Image Enhancement Factor (IEF) values but by time complexity theory it is time consuming and also it does not preserve edges and lines in the image. In the most of the existing work effort are made to remove impulse noise (salt and pepper noise).

In near future we will propose a new integrated method which will decrease the time complexity by using the Lossy Discrete Cosine Transform (DCT) compression technique and also it suppresses the impulse noise with higher degree of edge preservation by combining the hybrid median filter [2] and relaxed median filter [10].

\section{REFERENCES}

[1] S. Esakkirajan, T. Veerakumar, Adabala N. Subramanyam, and C. H. PremChand. 2011 Removal of High Density Salt and Pepper Noise Through Modified Decision Based Unsymmetric Trimmed Median Filter. IEEE SIGNAL PROCESSING LETTERS, VOL. 18, NO. 5

[2] Priyanka Kamboj, Versha Rani. 2013 Image Enhancement Using Hybrid Filtering Techniques. International Journal of Science and Research. Vol 2, No. 6, June 2013.

[3] Shanmugavadivu, Eliahim Jeevaraj. 2012 Laplace Equation based Adaptive Median Filter for Highly Corrupted Images. International Conference on Computer Communication and Informatics

[4] Shanmugavadivu P and Eliahim Jeevaraj P S. 2011 Fixed-Value Impulse Noise Suppression for Images using PDE based Adaptive Two-Stage Median Filter. ICCCET-11 (IEEE Explore), pp. 290-295.

[5] K. S. Srinivasan and D. Ebenezer. 2007 A new fast and efficient decision based algorithm for removal of high density impulse noise. IEEE Signal Process. Lett, vol. 14, no. 3, pp. 189-192

[6] V. Jayaraj and D. Ebenezer. $2010 \mathrm{~A}$ new switching-based median filtering scheme and algorithm for removal of high-density salt and pepper noise in image. EURASIP J. Adv. Signal Process.

[7] K. Aiswarya, V. Jayaraj, and D. Ebenezer. 2010 A new and efficient algorithm for the removal of high density salt and pepper noise in images and videos. Second Int. Conf. Computer Modeling and Simulation, pp. 409-413.

[8] Gnanambal llango and R. Marudhachalam. 2011 new hybrid filtering techniques for removal of Gaussian noise from medical images.ARPN Journal of Engineering and Applied Sciences.

[9] P. E. Ng and K. K. Ma. 2006 A switching median filter with boundary discriminative noise detection for extremely corrupted images. IEEE Trans. Image Process. vol. 15, no. 6, pp. 1506-1516

[10] Afrose. 2011 Relaxed Median Filter: A Better Noise Removal Filter for Compound Images. International Journal on Computer Science and Engineering (IJCSE) Vol. 4 No. 07

[11] Rafael C. Gonzalez, et al., 2005. Digital Image Processing using MATLAB, second Ed, Pearson Education, India.

[12] Median Filtering, [Last Visited] 18 June 2013 [Online] [Available] www.mathworks.com.

[13] Chan, R. H. Salt and pepper noise removal by median-type noise detectors and detail-preserving regularization.

IEEE Trans. on Image Processing, Vol. 14, no. 10, pp 1479-148

[14] H. Hwang and R. A. Hadded. 1995 Adaptive median filter: New algorithms and results. IEEE Trans. Image Process, vol. 4, no. 4, pp. 499-502, Apr. 1995.

[15] J. Astola and P. Kuosmaneen.1997 Fundamentals of Nonlinear Digital Filtering. Boca Raton, FL: CRC, 1997.

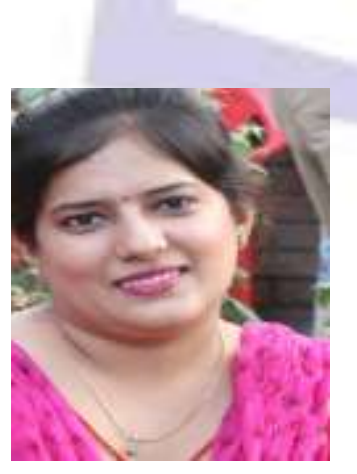

Sakshi Mahajan ,Student of Mtech,Final Year.Working as Assistant Professor in Department of Information Technology,Amritsar College of Engineering and Technology,Amritsar,Punjab.Her area of interest are image restoration and image enhancement. 